\title{
Disseminated Mycobacterium Avium Complex Mimicking Multi-Organ Sarcoidosis in a Patient with Systemic Lupus Erythematosus Treated with Rituximab
}

\section{ISSN: 2639-0531}

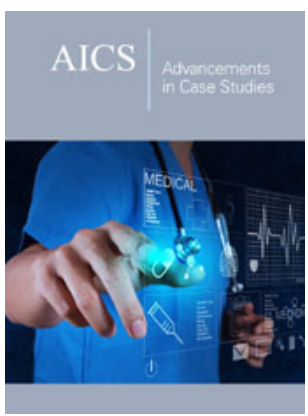

*Corresponding author: Tabata MM, Stanford University School of Medicine, USA

Submission: 战 September 02, 2019

Published: 眥September 20, 2019

Volume 2 - Issue 2

How to cite this article: Tabata MM Brian Abe and Shah NS. Disseminated Mycobacterium Avium Complex Mimicking Multi-Organ Sarcoidosis in a Patient with Systemic Lupus Erythematosus Treated with Rituximab. Adv Case Stud.2(1). AICS.000531.2019.

Copyright@ Tabata MM, This article is distributed under the terms of the Creative Commons Attribution 4.0 International License, which permits unrestricted use and redistribution provided that the original author and source are credited.

\author{
Tabata $\mathbf{M I M}^{1 *}$, Brian $\mathrm{Abe}^{2}$ and Shah $\mathrm{NS}^{2}$ \\ ${ }^{1}$ School of Medicine, USA \\ ${ }^{2}$ Department of Medicine, USA
}

\begin{abstract}
A 38-year old female with a history of systemic lupus erythematosus on rituximab therapy, bipolar disorder, renal dysfunction, and recurrent nephrolithiasis, presented to the hospital with fevers, flank pain, 40 -pound weight loss, odynophagia, and dysphagia. She was found to have new pancytopenia, pulmonary nodules and ground glass opacities on chest CT, and low-grade disseminated intravascular coagulation. She was treated with broad spectrum anti-microbials without improvement, and when her respiratory status rapidly declined, she was empirically started on steroids and quickly improved. After extensive workup, her overall clinical picture supported the diagnosis of sarcoidosis with pulmonary nodules, alkaline phosphatase elevation, hypercalcemia, elevated angiotensin converting enzyme and soluble IL-2 receptor level, and non-necrotizing granulomas on liver biopsy. Shortly after discharge, polymerase chain reaction of respiratory tract and blood cultures taken during hospitalization resulted positive for mycobacterium avium complex, consistent with disseminated Mycobacterium avium complex infection mimicking multi-organ system sarcoidosis.
\end{abstract}

Keywords: Sarcoidosis; Disseminated Mycobacterium avium complex; Systemic lupus erythematosus; Immunocompromised; Acute respiratory distress

Abbreviations: ACE: Angiotensin Converting Enzyme; ANA: Anti-Nuclear Antibody; BAL: Bronchoalveolar Lavage; CT: Computed Tomography; DIC: Disseminated Intravascular Coagulation; DMAC: Disseminated Mycobacterium Avium Complex; GGOs: Ground Glass Opacities; HLH: Hemophagocytic Lymphohistiocytosis; MLO: Mycoplasma-Like Organism; SLE: Systemic Lupus Erythematosus; PCR: Polymerase Chain Reaction

\section{Introduction}

This case demonstrates diagnostic challenges and overlapping features of disseminated Mycobacterium avium complex (DMAC) and multi-organ sarcoidosis. Mycobacterium complex are ubiquitous free-living organisms found in the natural reservoirs and transmitted to humans from the environment. Infection may be limited to pulmonary disease, and disseminated infection is typically seen in immunocompromised patients, such as those with HIV/AIDS. DMAC infection can manifest as fever, fatigue, weight loss, lymphadenopathy, splenomegaly, and involve many organ systems including the eye, musculoskeletal system, and skin. Laboratory tests for DMAC often reveal elevated inflammatory indices, cytopenias, and elevated creatinine. Blood cultures or bone marrow cultures should be obtained for when there is clinical suspicion of this syndrome [1].

Similarly, sarcoidosis may be limited to focal disease or can involve any organ system, most commonly the lungs, skin, eyes, liver, peripheral lymph nodes, heart, and bones. The etiology of sarcoidosis is unknown. Common findings include bilateral hilar adenopathy, pulmonary reticular opacities, nodular, papular or plaque-like lesions, erythema nodosum, migratory polyarthralgia, and fever. Pulmonary fibrosis can occur slowly and result in respiratory failure, pulmonary hypertension, and hemoptysis. Small fiber neuropathies and fatigue syndromes are common [1]. The findings of sarcoidosis can be variable, and diagnosis often requires a stepwise approach. Biopsy may show noncaseating granulomas. Lab tests may reveal elevated c-reactive protein, angiotensin converting enzyme, and soluble interleukin-2 receptor, but the utility of these tests is debatable. 


\section{Case Presentation}

A 38 year-old Caucasian female with a history of systemic lupus erythematosus (SLE), bipolar disorder, asthma, gastric sleeve, and recurrent nephrolithiasis presented to an outside hospital with three days of fevers up to 104 degrees Fahrenheit with associated chills and ten days of worsening bilateral flank pain, more severe on the right, with dysuria and hematuria. Urosepsis secondary to obstructing nephrolithiasis, seen on non-contrast computed tomography (CT) abdomen, was suspected, although no hydronephrosis was present. Additionally, test results were significant for leukopenia, thrombocytopenia, elevated alkaline phosphatase, and urinalysis showing $1+$ protein and 3+ blood (Table 1). Cultures from blood and urine were negative. At the outside hospital, the patient was started on cefepime, fluconazole, vitamin $\mathrm{K}$, and fresh frozen plasma without improvement. She was transferred to our hospital for a higher level of care, where additional review of systems included dry eyes, intermittent abdominal pain, pruritis over the arms and scalp, oral ulcers, shortness of breath, and arthralgias of bilateral hands. There was no Raynaud's phenomenon nor skin thickening. Notably, the patient had a history of SLE and was on rituximab infusions every six months, her last infusion five days prior to admission to the outside hospital. Also, in the five months prior to presentation, the patient reported a 40-pound unintentional weight loss due to poor PO intake secondary to dysphagia and odynophagia from esophageal candidiasis found on endoscopic biopsy.

Table 1: Laboratory values from outside hospital.

\begin{tabular}{|c|c|c|}
\hline Lab Test & Value & Reference Range \\
\hline WBC & 1.7 & $11.7-15.7 \mathrm{~g} / \mathrm{dL}$ \\
\hline Hemoglobin & 10.6 & $150-400 \mathrm{~K} / \mathrm{uL}$ \\
\hline Platelet count & 111 & $0.9-1.1$ \\
\hline INR & 2.5 & $22-34 \mathrm{sec}$ - \\
\hline Partial thromboplastic time & 59 & $<500 \mathrm{ng} / \mathrm{mL}$ \\
\hline D-dimer & 285 & $0.51-0.95 \mathrm{mg} / \mathrm{dL}$ \\
\hline Creatinine & 1.3 (baseline $0.8-0.9)$ & $3.5-5.2 \mathrm{~g} / \mathrm{dL}$ \\
\hline Albumin & 2.1 & $6-9 \mathrm{~g} / \mathrm{dL}$ \\
\hline Total protein & 4.0 & $35-105 \mathrm{U} / \mathrm{L}$ \\
\hline Alkaline Phosphatase & 744 & $10-35 \mathrm{U} / \mathrm{L}$ \\
\hline Aspartate transaminase & 48 & $<0.9 \mathrm{mg} / \mathrm{dL}$ \\
\hline C-reactive Protein & 28.5 & $2-20 \mathrm{~mm} / \mathrm{hr}$ \\
\hline Erythrocyte sedimentation rate & 3 & \\
\hline Urinalysis & Leukocyte esterase negative, $1+$ protein, 3+ blood, $22 \mathrm{WBC}$, & \\
\hline Urine Culture & 96 RBC & \\
\hline AFB Blood culture & $25,000-50,000$ mixed urogenital flora & \\
\hline
\end{tabular}

Table abbreviations: AFB: Acid Fast Bacilli; INR: International Normalized Ratio; RBC: Red Blood Cell; WBC: White Blood Cell

The patient first reported symptoms suggestive of SLE while in college with severe fatigue, rash, alopecia, shortness of breath, depression and anxiety. After delivery of her first child with an atrial septal defect, she was diagnosed with SLE with a reportedly positive ANA test at 1:2560 and skin biopsy showing urticarial vasculitis. However, this ANA test could not be confirmed by searching her medical records and contacting her past providers. She met additional criteria for SLE with malar rash, photosensitivity, oral lesions, and arthralgia. She was wellcontrolled with hydroxychloroquine for many years, requiring intermittent prednisone tapers for fatigue. Belimumab was added to her medication regimen one to two years prior for persistent fatigue. She began rituximab infusions one-year prior for continued shortness of breath, extreme fatigue, and arthralgia $(1000 \mathrm{mg}$ infusions twice, spaced 2 weeks apart, repeated every six months) and noted improved joint pain and energy. She completed the third dose $(1000 \mathrm{mg})$ of the fourth cycle five days prior to her current presentation. It should be noted that repeat ANA studies were performed to confirm the diagnosis of SLE. However, these were repeatedly negative by ELISA and indirect immunofluorescence after starting rituximab.

Seven months prior to current presentation, she presented to an outside hospital for persistent shortness of breath. Chest CT with contrast showed diffuse emphysematous changes with coarse bilateral interstitial markings and scattered patchy ground glass opacities (GGOs) concerning for an infectious or inflammatory process. She improved with antibiotics and 500mg of IV solumedrol, followed by $1 \mathrm{mg} / \mathrm{kg}$ of oral prednisone daily for two weeks after discharge. At discharge, the running diagnosis was sarcoidosis and consultation with internal medicine, rheumatology, and infectious disease teams favored an inflammatory process. 
Other past medical history included fibromyalgia and polycystic ovarian syndrome. Past surgical history includes cholecystectomy, two Cesarean sections, three inguinal hernia repairs, multiple lithotripsies, and bilateral tubal ligation. Home medications at the time of presentation included rituximab, hydroxychloroquine, metformin, omeprazole, venlafaxine, lithium, clonazepam, nadolol, promethazine, and acetaminophen/hydrocodone. Family history was significant for rheumatoid arthritis in her mother. The patient was a non-drinker and non-smoker and was on disability for the past seven years. At our hospital, the patient had an oxygen saturation of $95 \%$ on 2 liters nasal cannula. Physical exam was significant for somnolence, temporal wasting, xerostomia, costovertebral angle tenderness, and $2+$ bilateral pitting edema. There was no malar or discoid rash.

Initial laboratory results at our hospital revealed thrombocytopenia, anemia, leukopenia, low total protein, and elevated creatinine, alkaline phosphatase, international normalized ratio, and c-reactive protein (Table 2). D-dimer was elevated at 412 and fibrinogen was low at 32. Additional workup revealed negative results for ANA (1:80 dilution, Hep2 substrate), anti-ds DNA, anti-Ro, anti-La, anti-Smith, p-ANCA, c-ANCA, proteinase3, myeloperoxidase, glomerular basement membrane, and free kappa and lambda light chain. IgA, IgG, and IgM were low at values of 55, 504, and 9.31, respectively. CD4, CD8, and NK counts were low at 225, 143, and 33 respectively. B-cell count was zero. Angiotensin converting enzyme (ACE) was elevated at 129. Corrected calcium was elevated at 10.3 , and 25-hydroxy vitamin $\mathrm{D}$ and parathyroid hormone were low at $8.8,11$, and $<3$, respectively. Parathyroid hormone-related peptide was within normal limits. Ferritin was elevated to 250 on repeat tests.

Table 2: Laboratory studies on initial presentation at our hospital.

\begin{tabular}{|c|c|c|}
\hline Test & Result value & Reference value \\
\hline Sodium & 138 & $135-145 \mathrm{mmol} / \mathrm{L}$ \\
\hline Potassium & 3.6 & $3.5-5.3 \mathrm{mmol} / \mathrm{L}$ \\
\hline Chloride & 109 & $98-110 \mathrm{mmol} / \mathrm{L}$ \\
\hline Bicarbonate & 23 & $20-31 \mathrm{mmol} / \mathrm{L}$ \\
\hline Blood urea nitrogen & 12 & $7-25 \mathrm{mg} / \mathrm{dL}$ \\
\hline Creatinine & 1.2 (baseline $0.8-1.0)$ & $0.51-0.95 \mathrm{mg} / \mathrm{dL}$ \\
\hline Glucose & 105 & $70-100 \mathrm{mg} / \mathrm{dL}$ \\
\hline WBC & 1.4 & $4-11 \mathrm{~K} / \mathrm{uL}$ \\
\hline Hemoglobin & 9.0 & $11.7-15.7 \mathrm{~g} / \mathrm{dL}$ \\
\hline Hematocrit & 27.4 & \\
\hline $\begin{array}{c}\text { Urinalysis } \\
\text { Complement C3 }\end{array}$ & $21-50 \mathrm{RBC}$ & \\
\hline $\begin{array}{c}\text { Complement C4 } \\
\text { Alanine } \\
\text { transaminase }\end{array}$ & 41.8 (normal) & $20-59 \mathrm{mg} / \mathrm{dL}$ \\
\hline $\begin{array}{c}\text { Aspartate } \\
\text { transaminase }\end{array}$ & 43 & $6-29 \mathrm{U} / \mathrm{L}$ \\
\hline & 34 & $10-35 \mathrm{U} / \mathrm{L}$ \\
\hline
\end{tabular}

\begin{tabular}{|c|c|c|}
\hline $\begin{array}{c}\text { Alkaline } \\
\text { phosphatase }\end{array}$ & 793 & $35-105 \mathrm{U} / \mathrm{L}$ \\
\hline Total bilirubin & 1.3 & $<1.4 \mathrm{mg} / \mathrm{dL}$ \\
\hline Albumin & 2.0 & $3.5-5.2 \mathrm{~g} / \mathrm{dL}$ \\
\hline Total protein & 3.8 & $6-9 \mathrm{~g} / \mathrm{dL}$ \\
\hline Globulin & 1.8 & $2-5 \mathrm{~g} / \mathrm{dL}$ \\
\hline INR & 2.6 & $0.9-1.1$ \\
\hline $\begin{array}{c}\text { Erythrocyte } \\
\text { sedimentation rate }\end{array}$ & 2 & $2-20 \mathrm{~mm} / \mathrm{hr}$ \\
\hline C-reactive protein & 3.3 & $<0.9 \mathrm{mg} / \mathrm{dL}$ \\
\hline
\end{tabular}

Table abbreviations: INR: International Normalized Ratio; RBC: Red Blood Cell; WBC: White Blood Cell

Blood tests and bronchoalveolar lavage (BAL) studies revealed negative workup for coccidiodes, histoplasma, pneumocystis jiroveci pneumonia, acid fast bacilli, legionella, brucella, coxiella burnetti, strongyloides, toxoplasma, human Immunodeficiency virus, cytomegalovirus, Ebstein-Barr virus, parvovirus B19, influenza $A$ and $B$, respiratory syncytial virus, parainfluenza 1-4, metapneumovirus, rhinovirus, enterovirus, adenovirus, coronavirus, chlamydia pneumoniae, and mycoplasma pneumoniae. Viral, bacterial and fungal culture and polymerase chain reaction (PCR) were negative. Fungi-tell beta-D glucan assay was positive. Aspergillus galactomannan was negative from blood but was elevated in BAL studies.

CT of the chest, abdomen, and pelvis revealed nonspecific pulmonary findings of small bilateral pleural effusions, intralobular septal thickening and scattered GGOs. Wall thickening of the cecum and ascending colon was concerning for nonspecific colitis, and mesenteric stranding and edema involving the pancreas was concerning for pancreatitis. Splenomegaly was noted. Subsequent whole-body FDG-PET CT showed hypermetabolic nodular pulmonary opacities, mildly enlarged mediastinal and hilar lymphadenopathy without FDG uptake. Bone biopsy showed hemophagocytosis, but there was no abnormal hypermetabolic activity in the bone marrow to suggest hemophagocytic lymphohistiocytosis (HLH). Brain MRI was unremarkable.

The patient became more somnolent throughout the first week of admission and labs consistent with disseminated intravascular coagulation (DIC) worsened. D-dimer increased steadily from 412 on admission to greater than 12,000 and fibrinogen continued to drop, requiring repeated, almost-daily cryoprecipitate infusions. The patient developed worsening crackles and ground-glass opacities throughout the first week, and on day 8 the patient experienced fever of 101F, hemoptysis, and respiratory distress requiring 6 liters of oxygen through a high flow mask. At this time the patient was on doxycycline $100 \mathrm{mg}$ daily and voriconazole $4 \mathrm{mg} / \mathrm{kg}$ IV every 12 hours. The patient was given pulse methylprednisolone as infectious workup was negative and suspicion for an autoimmune cause of her acute respiratory failure was high, and she rapidly improved to breathing on room air within two days. Alkaline phosphatase elevation, hypercalcemia, d-dimer, and INR resolved with steroid use. 
Bone marrow biopsy showed a decrease in cellularity but no atypical lymphoid infiltrates, increased blasts, viral inclusions, or granulomas. Bone marrow aspirate showed occasional histiocytes with ingested red blood cells with no increase in hemophagocytosis of nucleated cells. Liver biopsy showed macro-vesicular steatosis and non-necrotizing portal and lobular granulomas. Soluble IL-2 receptor was elevated at 27,600 (reference $<1,000$ ).

The patient was treated with broad spectrum antibiotics and antifungals, including vancomycin, doxycycline, levofloxacin, piperacillin/tazobactam, cefepime, fluconazole, and voriconazole throughout the hospitalization. After the episode of acute respiratory distress, she completed three days of IV methylprednisolone (500mg) and was maintained on prednisone $(1 \mathrm{mg} / \mathrm{kg})$, as well as voriconazole for aspergillus and atovaquone for empiric coverage of pneumocystis carinii pneumonia (PCP). Hypercalcemia resolved after administering zoledronic acid.

The principal diagnosis at hospital discharge was multiorgan sarcoidosis with pulmonary, hepatic, renal and bone marrow involvement, supported by elevated ACE, soluble IL-2R, nonnecrotizing granulomas on liver biopsy, and rapid response to steroids. The patient's odynophagia was thought to be caused by oral and esophageal candidiasis in the setting of severe immunosuppression, which resolved after empiric treatment. The patient continued to have elevated D-dimer, prothrombin time, partial thromboplastin time and low fibrinogen after steroid treatment, despite negative blood tests for aspergillus galacatomannan. She was treated for possible pulmonary aspergillus with voriconazole given the elevated BAL aspergillus galactomannan studies and GGO on CT chest.

Less than one month after hospital discharge, PCR results from respiratory and blood cultures sampled during hospitalization on two separate consecutive days resulted positive for MAC. In light of these new results, her multi-organ failure was considered to be secondary to disseminated MAC, rather than sarcoidosis. Her steroids were tapered, and she began treatment with azithromycin, ethambutol and rifabutin, resulting in considerable improvement.

\section{Discussion}

This was a challenging case of disseminated MAC presenting with laboratory and biopsy findings suggestive of multi-organ sarcoidosis. To date, there are no published cases of disseminated MAC mimicking multi-organ system sarcoidosis, although there have been two cases of cutaneous MAC resembling sarcoidosis [2]. Interestingly, a mycoplasma-like organism (MLO) was postulated to be a cause of sarcoidosis in 1996, and inoculation of MLO into mouse eyelids produced chronic uveitis and lethal systemic granulomatous disease [3]. In 1995 two immunocompetent patients with cutaneous granulomatous lesions resembling sarcoidosis were reported to have cultures positive for Mycobacterium avium intracellular [2]. Recent meta-analysis and review found a significant association between sarcoidosis and mycobacteria, with an OR of 6.8 (95\% CI 3.73, 12.39), although the mechanistic explanation for this relationship remains controversial [4]
DMAC occurs almost exclusively in late-stage AIDS patients [1]. Non-AIDS patients most often have an underlying immunodeficiency, and approximately $80 \%$ of genetically diagnosed cases are caused by IFN- $\gamma$ receptor or IL-12 receptor deficiency [1]. DMAC infection has been reported in a 35-year-old immunocompetent female, manifesting as hepatic granulomas [5]. It has been postulated that exposure to unusually high doses of mycobacterium may lead to disease in immunocompetent patients [6]. High doses of inhaled corticosteroid are associated with non-tuberculosis mycobacterium, possibly due to underlying lung disease requiring steroid treatment [7]. Although DMAC is not classically associated with DIC, chronic DIC has been reported in a 33-year-old AIDS patient who presented with nonspecific symptoms, anemia, thrombocytopenia, increased lactate dehydrogenase, alkaline phosphatase, and ferritin, and diagnosed with DMAC on bone marrow biopsy and blood and sputum cultures [8].

In our patient, we believe that immunosuppression with rituximab likely contributed to DMAC infection. DIC may have been a consequence of MAC. While there is always a possibility of contamination of PCR leading to a false positive, multiple positive test separated in time and improvement with treatment increases the likelihood of this being a true infection. Rapid pulmonary improvement with methylprednisolone during her hospitalization is believed to be due to treatment of the inflammatory component of DMAC, similar to immune reconstitution and inflammatory syndrome. Another diagnostic consideration that mimics DIC is catastrophic antiphospholipid syndrome, a severe variant of antiphospholipid syndrome characterized by disseminated microangiopathy that results in multiorgan failure and mainly occurs in association with SLE [9]. However, our patient did not experience vascular thromboses consistent with catastrophic antiphospholipid syndrome. Additionally, our patient's elevated ferritin raised suspicion for HLH, and although rare, Gupta et. al. reported two cases of secondary HLH with SLE. Both have overlapping clinical features, but unlike SLE, HLH is characterized by hyperferritinemia, hypofibrinogenemia, hypertriglyceridemia, and a decrease in erythrocyte sedimentation rate (ESR) and NK cell activity [10]. HLH is not typically associated with MAC, although MAC has been reported as the cause of HLH in a patient with acute lymphoblastic leukemia [11]. Although initial bone marrow biopsy raised suspicion for HLH, liver biopsy did not show hemophagocytosis consistent with HLH.

Our patient's persistently negative ANA test results raised the question of whether she truly had SLE. There is a small population of ANA negative SLE patients, with reports of up to 20 percent of these patients having an autoantibody against cellular components [12]. Additionally, we cannot rule out the possibility that her rituximab treatments may have affected her ANA titers, as B cell depletion therapy can result in negative ANA titers [13]. We relied on previous medical records regarding the presence of symptoms and immunologic data that was consistent with a diagnosis of SLE. Additionally, the known genetic association of SLE-induced sarcoidosis in African Americans gives rise to the question of 
screening individuals with SLE for a predisposition to develop sarcoidosis [14]. At this time, no screening exists, although there is evidence for different genetic predispositions for sarcoidosis in white and black patients [15].

In summary, a patient with a history of SLE presented with weight loss, respiratory distress, and renal failure and was found to have hilar adenopathy, pulmonary nodules, elevated alkaline phosphatase, calcemia, angiotensin converting enzyme and soluble IL-2 receptor, with liver biopsy showing non-necrotizing granulomas, suggestive of multi-organ sarcoidosis, but was ultimately diagnosed with disseminated MAC based on multiple positive PCR tests. In the complex rheumatologic patient, thorough workup must be performed for all possible infections and coexisting immunologic diseases given the overlapping clinical features. It is important to recognize clinical similarities of DMAC and multi-organ sarcoidosis, consider the possibility of DMAC mimicking sarcoidosis in an immunocompromised patient, and search for an underlying immunodeficiency in a non-AIDS patient who presents with DMAC.

\section{Acknowledgements}

MMT contributed to patient care, manuscript writing, and manuscript editing.

BA contributed to patient care and manuscript editing.

NSS contributed to patient care and manuscript editing.

\section{References}

1. Daley CL (2017) Mycobacterium avium complex disease. Microbiol Spectrum 5(2): TNMI7-0045.

2. Epps RE, El-Azhary RA, Hellinger WC, Winkelmann RK, Van Scoy RE (1995) Disseminated cutaneous Mycobacterium avium-intracellular resembling sarcoidosis. J Am Acad Dermatol 33(3): 528-531.

3. Johnson LA, Edsall JR, Austin JH, Ellis K (1996) Pulmonary sarcoidosis: could mycoplasma-like organisms be a cause? Sarcoidosis Vasc Diffuse Lung Dis 13(1): 38-42.

4. Esteves T, Aparicio G, Garcia-Patos V (2016) Is there any association between Sarcoidosis and infectious agents?: a systematic review and meta-analysis. BMC Pulm Med 16(1): 165.
5. Yabes JM, Farmer A, Vento T (2017) Disseminated Mycobacterium avium complex in an immunocompetent host. Int J Mycobacteriol 6(2): 202206.

6. Dirac MA, Horan KL, Doody DR, Meschke JS, Park DR, et al. (2012) Environment or host? A case-control study of risk factors for Mycobacterium avium complex lung disease. Am J Respir Crit Care Med 186(7): 684-691.

7. Hojo M, Iikura M, Hirano S, Sugiyama H, Kobayashi N, et al. (2012) Increased risk of nontuberculous mycobacterial infection in asthmatic patients using long-term inhaled corticosteroid therapy. Respirology 17(1): 185-190.

8. Ayoade F, Cotelingam J, Joel Chandranesan AS (2017) Disseminated mycobacterium avium-intracellular complex infection presenting with disseminated intravascular coagulation in an aid's patient. Journal of Investigative Medicine High Impact Case Reports 5(4): 2324709617740904 .

9. Mizuno R, Fujimoto S, Fujimoto T, Nishino T, Shiiki H, et al. (2012) Catastrophic antiphospholipid antibody syndrome in systemic lupus erythematosus: an autopsy case report of a young woman. Internal Medicine 39(10): 856-859.

10. Gupta D, Mohanty S, Thakral D, Bagga A, Wig N, et al. (2016) Unusual association of hemophagocytic lymphohistiocytosis in systemic lupus erythematosus: Cases reported at tertiary care center. The American Journal of Case Reports 17: 739-744.

11. Ordaya EE, Jarir SA, Yoo R, Chandrasekar PH (2017) Hemophagocytic lymphohistiocytosis (HLH): Elusive diagnosis of disseminated Mycobacterium avium complex infection. Germs 7(3): 149-152.

12. Choi MY, Clarke AE, St Pierre Y, Hanly JG, Urowitz MB, et. al. (2019) Antinuclear antibody-negative systemic lupus erythematosus in an international inception cohort. Arthritis Care Res 71(7): 893-902.

13. Rheumatol J (2016) Specific antinuclear antibody level changes after b cell depletion therapy in systemic sclerosis are associated with improvement of skin thickening. J Rheumatol 43(3): 681.

14. Levin AM, Adrianto I, Datta I, Iannuzzi MC, Trudeau S, et al. (2015) Association of HLA-DRB1 with Sarcoidosis Susceptibility and Progression in African Americans. Am J Respir Cell Mol Biol 53(2): 206216.

15. Petit A (2013) Multisystemic diseases and ethnicity: a focus on lupus erythematosus, systemic sclerosis, sarcoidosis and Behçet disease. British Journal of Dermatology 3: 1-10. 